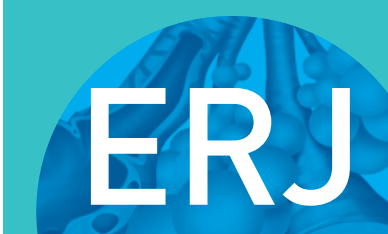

open research
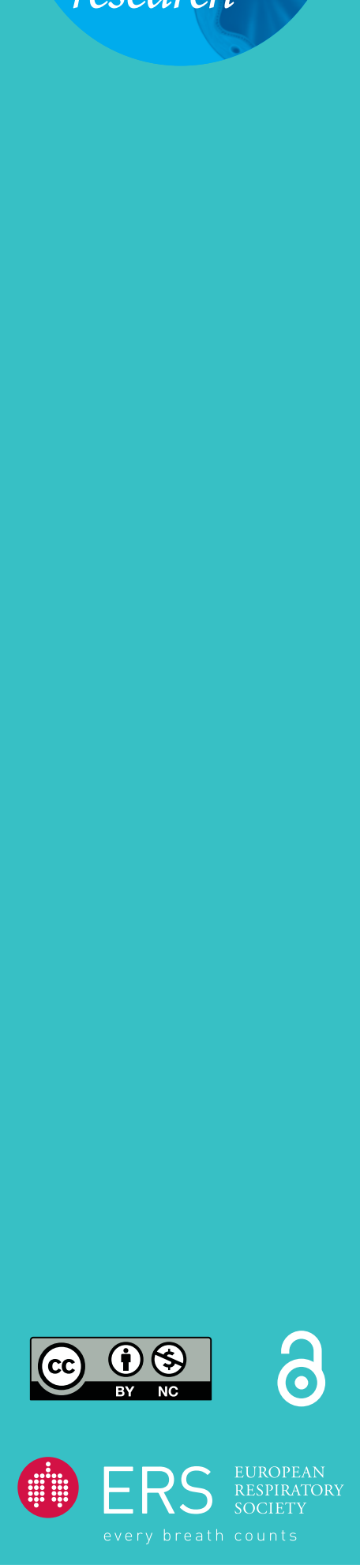

\section{The six-gene expression signature in whole sampled sputum provides clinically feasible inflammatory phenotyping of asthma}

\author{
Laurits Frøssing ${ }^{1,2}$, Ditte Kjærsgaard Klein ${ }^{1,2}$, Vibeke Backer ${ }^{3}$, Katherine \\ Joanne Baines ${ }^{4}$ and Celeste Porsbjerg ${ }^{1,2}$
}

Affiliations: ${ }^{1}$ Respiratory Research Unit, Bispebjerg University Hospital, Copenhagen, Denmark. ${ }^{2}$ Copenhagen Center for Translational Research, Bispebjerg University Hospital, Copenhagen, Denmark. ${ }^{3}$ Center for Physical Activity Research, Rigshospitalet, Copenhagen, Denmark. ${ }^{4}$ Respiratory and Sleep Medicine, University of Newcastle, Newcastle, Australia.

Correspondence: Laurits Frøssing, Respiratory Research Unit, Bispebjerg University Hospital, Bispebjerg Bakke 23, Copenhagen NV 2400, Denmark. E-mail: froessingladadlnet.dk

ABSTRACT Processing of induced sputum is time consuming and requires trained personnel, and consequently the use of induced sputum is limited to few sites globally. The six-gene signature (6GS) is an mRNA-based gene signature that was developed to provide a clinically feasible method for inflammatory phenotyping. In this study, we assessed whether the 6GS would perform similarly in induced sputum sampled using a simplified method, by which induced sputum can be sampled and stored directly for later qPCR analyses, to the conventional method of manual plug selection.

Two separate sputum samples were collected from 27 patients with asthma; one processed as a whole sample in an Oragene-RNA RE 100 vial and one processed using manual plug selection. Expression of 6GS was measured in both samples, of which 20 pairs $(74 \%)$ had enough samples and results of sufficient quality of gene expression for further analyses.

We found a significantly higher median RNA concentration in whole sampled sputum and consistently stronger gene expression compared to the plug method. Further, we found the two methods to agree, as $97 \%$ of observations were within the limits of agreement, as well as having a good-to-excellent reliability using intraclass correlation. Finally, we found 6GS in the whole sampled sputum to perform equal to or better than the manually selected plugs for discriminating inflammatory phenotypes defined by sputum differential count.

In conclusion, whole sampling of induced sputum provides a clinically feasible method for inflammatory phenotyping.

@ERSpublications

Collection of sputum using the whole sputum method in an RNA-stabilising kit obviates the need for immediate handling, and is comparable to the plug selection method with regard to mRNA expression and mRNA-based discrimination of inflammatory phenotypes http://bit.ly/ 2SqmBJ7

Cite this article as: Frøssing L, Kjærsgaard Klein D, Backer V, et al. The six-gene expression signature in whole sampled sputum provides clinically feasible inflammatory phenotyping of asthma. ERJ Open Res 2020; 6: 00280-2019 [https://doi.org/10.1183/23120541.00280-2019].

Received: 10 Oct 2019 | Accepted after revision: 17 Dec 2019

Copyright OERS 2020. This version is distributed under the terms of the Creative Commons Attribution NonCommercial Licence 4.0. 


\section{Introduction}

Induced sputum is the gold standard for assessing the pattern of airway inflammation in asthma and its use for inflammatory phenotyping is advocated in the European Respiratory Society (ERS)/American Thoracic Society (ATS) guidelines on severe asthma [1].

Since the first standardised protocols for sampling induced sputum, two methods of processing have evolved. The "plug selection method" involves selection of all viscid or denser parts of the sample, while the entire sample, including variable amounts of saliva, is processed using the "whole sputum method" $[2,3]$. Both collection methods have been found to yield similar results for cell differential count, however no studies have compared the two methods with regards to mRNA expression [4].

The six-gene expression signature (6GS) is an mRNA-based gene signature that was developed as a molecular diagnostic tool to provide a clinically feasible method for inflammatory phenotyping of asthma; however, implementation in clinical practice has been hampered by the immediate requirements of processing induced sputum to stabilise RNA for gene expression analyses [5].

Easy-to-use mRNA-stabilising kits are commercially available and potentially offer huge advantages over the current processing protocols as they provide stabilisation of the sample upon collection (using an RNA-stabilising buffer), thereby removing the immediate need for sample processing. However, whether these kits can allow accurate reflection of airway inflammation is unknown. Further, in contrast to the "plug selection method" employed by most groups assessing mRNA expression in induced sputum [5-7], these kits rely on sampling sputum using the "whole sputum method" and the implications of increased contamination with salivary cells on RNA composition and the diagnostic utility of the sample is unknown $[2,3]$.

We believe validation of an easy-to-use kit providing immediate mRNA stabilising is key to developing a clinically feasible method for inflammatory phenotyping and biobanking outside highly specialised centres. Therefore, the aim of this study was to compare 6GS gene expression measured on sputum collected using the "whole sample method" in an mRNA-stabilising kit with sputum collected using the "plug selection method".

Further, to assess whether this new methodology accurately reflected airway inflammation, we wanted to compare the ability of the two collection methods to discriminate inflammatory phenotypes of asthma using expression of the 6GS. We hypothesised that sputum collected using the "whole sample method" in an mRNA-stabilising kit was non-inferior to sputum collected using the "plug selection method" with regards to RNA yield, levels of gene expression of $6 \mathrm{GS}$ and the ability of 6GS to discriminate inflammatory phenotypes of asthma.

\section{Methods}

Two separate sputum samples were collected from 27 asthma patients referred for evaluation of asthma at the Respiratory Outpatient Clinic at Bispebjerg Hospital, Denmark, who all participated in the BREATHE (Biomarker Research and E-Health Advancements To Help Everyone) study (ethical approval: H-16047428).

Criteria for participation were respiratory symptoms (e.g. dyspnoea, cough, wheezing or chest tightness) and 1) either a current positive bronchial challenge test or 2) a doctor's diagnosis of asthma and a current asthma maintenance therapy corresponding to step 4 or 5 in the 2017 Global Initiative for Asthma guidelines.

One sputum sample was collected in an Oragene-RNA RE 100-vial (DNA Genotek; Ontario, Canada) using the "whole sputum method" and one sputum sample was collected in a Petri dish and processed using the "plug selection method". The Oragene-RNA RE 100 kit is commercially available and has been developed for collection and preservation of mRNA in saliva for research purposes. The vial can contain a sample of up to $2 \mathrm{~mL}$ and holds the sample stable at room temperature for 60 days.

\section{Sputum induction}

Sputum was collected after a bronchial challenge test with mannitol. Sputum was induced using isotonic saline if forced expiratory volume in $1 \mathrm{~s}\left(\mathrm{FEV}_{1}\right)$ was $<70 \%$ of predicted [8].

The mannitol challenge involved inhalation of incremental doses of mannitol and the patients were instructed to cough and expectorate sputum after each inhalation. Spirometry was measured 1 min after each dose and the test was completed when a $15 \%$ decline from baseline $\mathrm{FEV}_{1}$ was recorded or when reaching a cumulative dose of $635 \mathrm{mg}[9,10]$.

\section{Sputum handling}

Both sputum samples were collected on the same day and within the same induction. First, the patient provided a sample for the "plug selection method", which was collected in a specimen jar (Petri dish). 
This sample was continuously evaluated by a trained lab technician and when considered to be of sufficient size and quality, all subsequent expectorated sample was collected using the "whole sputum method" in the Oragene-RNA RE 100-vial kit.

\section{Handling using the "plug selection method"}

The sputum sample was processed within $1 \mathrm{~h}$ of collection as described by BAFADHEL et al. [11] and a minimum of two plugs were selected depending on the quantity of the sputum sample: one for cell differential count (CDC), which was dispersed with dithiothreitol and one stored in RLT buffer and frozen at $-80^{\circ} \mathrm{C}$ for later gene expression analysis. When restricted by sample quantity, the CDC was prioritised.

\section{Handling using the "whole sputum method"}

The entire sample was collected using the "whole sputum method" in an Oragene-RNA RE-100 kit as per manufacturer's instructions (DNA Genotek, Ottawa, Canada.). Upon closure of the vial cap, a non-specified stabilising liquid was released from the cap and the capped vial was shaken for $10 \mathrm{~s}$. No additional handling or processing was done, and the sample was stored at $-40^{\circ} \mathrm{C}$ for subsequent gene expression analysis.

\section{Sputum gene expression analyses}

The whole sputum collected in the Oragene-RNA RE 100-vial kit required several processing steps before RNA extraction in accordance with the accompanying protocol (DNA Genotek) [12]. This included an initial incubation at $50^{\circ} \mathrm{C}$ for $1 \mathrm{~h}$, addition of Oragene RNA neutraliser solution and cold 95\% ethanol, centrifugation and resuspension of the resulting pellet in RLT buffer.

RNA extraction of samples in RLT buffer, including a DNase step, was then performed using an automated method (QIAcube) with the RNeasy Mini Kit as per manufacturer's instructions and as previously published (QIAGEN; Hilden, Germany) [5]. RNA quantity was measured using the Nanodrop (Thermo Fisher, Waltham, USA). Up to $200 \mathrm{ng}$ RNA was converted to cDNA using reverse transcriptase, and 6GS expression (CLC, CPA3, DNASE1L3, IL1B, ALPL, CXCR2) was measured by qPCR using standard TaqMan methods. Expression of 6GS was assessed in each sample and the change $(\Delta)$ in cycle threshold (CT) for each gene was calculated relative to housekeeping gene $18 \mathrm{~s}$ [13].

RNA samples were considered adequate if the level of $18 \mathrm{~s}$ housekeeping gene expression was $<22 \mathrm{CT}$.

\section{Sputum cell differential count}

The sputum CDC was performed on a light microscope by an experienced lab technician at Bispebjerg University Hospital, Copenhagen, Denmark. Cell viability was evaluated using the Trypan blue exclusion method. Cut-off values for eosinophilia and neutrophilia were set to $\geqslant 3 \%$ and $>61 \%$, respectively, based on the CDC and patients were classified as eosinophilic if they had sputum eosinophils $\geqslant 3 \%$, and neutrophilic if their sputum neutrophils were $\geqslant 61 \%[5,11]$.

\section{Clinical assessments}

Atopy (allergic sensitisation) was defined as a specific $\operatorname{IgE}>0.35 \mathrm{kU} \cdot \mathrm{L}^{-1}$ for a minimum of 1 out of 10 tested aeroallergens [14]. Spirometry was conducted in accordance with ERS guidelines and a MasterScreenPneumo spirometer (BD Jaeger, Würzburg, Germany) was used for spirometry [15]. Predicted values were calculated with the use of the National Health and Nutrition Examination Survey reference data [16]. Exhaled nitrogen oxide fraction $\left(F_{\mathrm{ENO}}\right)$ measurements were done using the Niox Vero (Circassia Limited, Oxford, UK) [17].

Blood eosinophilia was assessed by the Bispebjerg Hospital pathology laboratory using automated flow cytometry with a Sysmex XN 9000 (Sysmex Europe GmbH, Norderstedt, Germany) and blood eosinophilia was defined as a blood eosinophil count $\geqslant 0.3 \times 10^{9} \cdot \mathrm{L}^{-1}$.

\section{Statistics}

SAS Enterprise guide version 7.1 (SAS Institute, Cary, NC, USA) was used for data analysis. Means (SD) are presented for normally distributed data and medians (quartile 1; quartile 3) are presented for non-parametric data.

Statistical analysis was done on the delta CT changes $\left(2^{-\Delta C T}\right)$ between the target gene of interest and the housekeeping gene $18 \mathrm{~s}$ [13].

The two methods were compared on expression level using intraclass correlation (ICC). ICC estimates were calculated by an absolute-agreement, two-way mixed-effects model $(\mathrm{k}=2)$. 
Subsequently the expression of each gene individually was evaluated using the Bland-Altman approach; a method specifically developed to describe agreement between two quantitative measurements using limits of agreement to quantify agreement [18]. We calculated these statistical limits using the logarithmic $\left(\log _{10}\right)$ $\Delta \mathrm{CT}$ changes $\left(2^{-\Delta \mathrm{CT}}\right)$.

Multiple logistic regression was used to calculate the predicted value of a subject having a given phenotype based on their level of expression for each method independently using the delta CT changes $\left(2^{-\Delta C T}\right)$ [5]. Receiver operating characteristic (ROC) curves were generated, and the area under the curve (AUC) was calculated. The respective ROC curves were compared using a Chi-squared test.

Classification of inflammation using gene expression was calculated from the multiple logistic regression models, where the optimal cut-off point for each ROC curve was calculated. We assessed two different methods for calculating the optimal cut-off point; one where the optimal cut-off point was defined as the point on the ROC curve with the minimum distance to the upper left corner (the Euclidian distance):

$$
d=\sqrt{\left(\left(1-\text { sensitivity }^{2}\right)+\left(1-\text { sensitivity }^{2}\right)\right)}
$$

where sensitivity $=1$ and specificity $=1$; and one defined as the point for which sensitivity and specificity is maximal (Youden index). We found identical cut-off points for all ROC curves using the two methods.

We then calculated the sensitivity, specificity, PPV and NPV for each method relative to the gold standard (CDC). Finally, we calculated Cohen's kappa coefficient for each method to evaluate the agreement in classification between the respective methods and the CDC classification.

\section{Results}

\section{Study population}

A total of 20 patients (74\%) were included in the analyses of gene expression, as 7 patient sample pairs were excluded due to insufficient RNA (determined as weak (>22)18 s CT housekeeping values; see figure 1)). One (5\%) patient had sputum collected after induction with isotonic saline, whereas all other patients had sputum collected after a mannitol challenge test. Patient characteristics are summarised in table 1 . The mean age was 39 years (SD 19) and 55\% of the patients were male. The mean $\mathrm{FEV}_{1} \%$ predicted was 93 (SD 20) and the median budesonide-equivalent inhaled corticosteroid dose was $800(600,800)$. Forty-five percent of the patients were atopic, while the median level of $\operatorname{IgE}$ was $62(20,217)$. The median level of blood eosinophils was $0.13(0.09,0.29)$, sputum eosinophil levels were $2.6(0.9,4.5)$, and $F_{\mathrm{ENO}}$ was median (interquartile range) $15(8,23)$. There were nine patients $(45 \%)$ with eosinophilic airway inflammation, five patients $(25 \%)$ with neutrophilic airway inflammation and three (15\%) of these who had both eosinophilic and neutrophilic inflammation.

\section{Expression of target genes}

We found a significantly $(\mathrm{p}<0.001)$ higher median RNA concentration in the whole sampled sputum (47.6 $(25.1,132.0)) \mathrm{ng} \cdot \mu \mathrm{L}^{-1}$ compared to the sputum collected using the "plug selection method" $\left(5.1 \mathrm{ng} \cdot \mu \mathrm{L}^{-1}\right.$

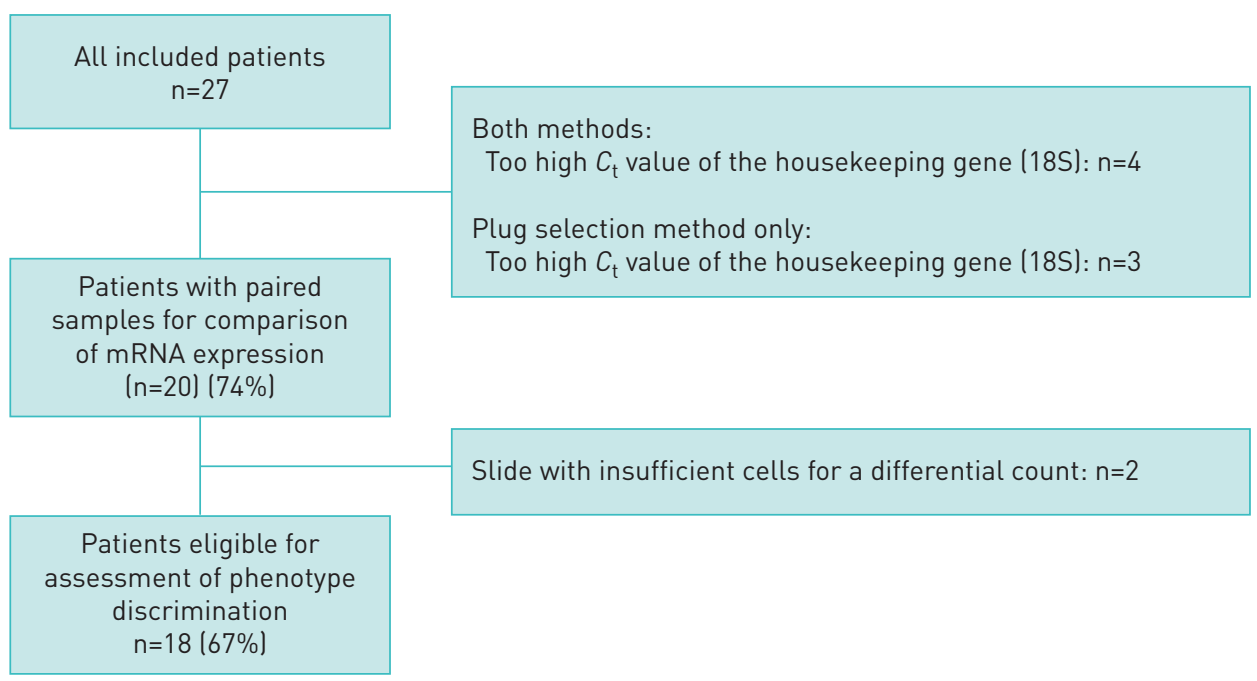

FIGURE 1 Flow chart describing the population of patients used for analysis. $C_{\mathrm{t}}$ : cycle threshold. 


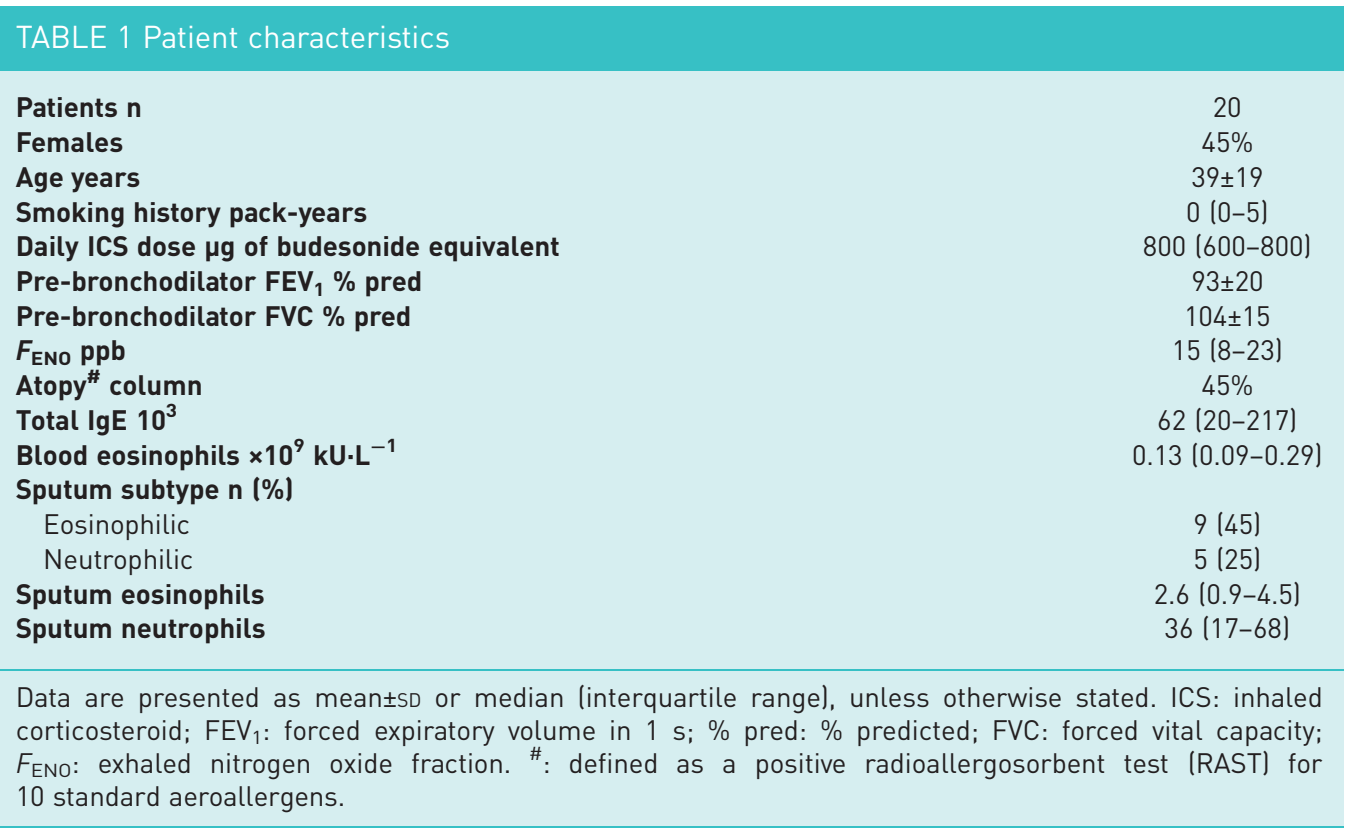

$(3.5,6.9))$. As listed in table 2 , median expression of all target genes was higher in the whole sampled sputum with the neutrophilic gene $A L P L$, and to a lesser extent the eosinophilic gene DNASE1L3, having markedly larger difference in expression as illustrated in table $2[5,19]$. For all genes, as well as for the RNA concentration, we found a good-to-excellent inter-method reliability as measured by ICC; the gene $(A L P L)$ with the biggest difference in expression also had the lowest ICC [20]

Using the Bland-Altman approach, we plotted the difference between the two methods against the mean as shown in figure 2. Across all assessed genes we found five (4\%) observations outside the limits of agreement (illustrated by the dashed red line in figure 2), which by convention is what is to be expected (mean of the difference $\pm 2 \times \mathrm{SD}$ ). The plot for CLC could reflect a trend towards increasing mean resulting in a decrease in difference, while the plot for $A L P L$ suggest that the lowest means were associated with the largest differences. We found no apparent patterns in the other plots.

\section{Prediction of inflammatory phenotypes using 6GS}

The CDC obtained from the manually selected plugs was used as the gold standard for classification of inflammatory phenotype (eosinophilic: $n=9$; neutrophilic: $n=5$ ). Sputum differential count was available for 18 of the 20 patients and patients without a CDC were excluded for the analysis of inflammatory phenotype prediction (figure 1).

Discriminating eosinophilic asthma from non-eosinophilic asthma

The 6GS resulting from the "plug selection method" (figure 3a; AUC 85\%, p=0.1) and "whole sputum method" (figure 3a; AUC 100\%, p=0.01) was able to discriminate patients with eosinophilic asthma

\section{TABLE 2 Comparison of RNA concentration and gene expression across methods}

\begin{tabular}{lccc} 
& Manually selected plugs & Whole sample sputum & Intraclass correlation \\
\hline RNA concentration $\mathrm{ng} \cdot \boldsymbol{\mu L}^{-1}$ & $5.10(3.5-6.9)$ & $47.6(25.1-132.0)$ & 0.93 \\
CLC expression & $0.09(0.01-0.33)$ & $0.15(0.07-0.54)$ & 0.84 \\
CPA3 expression & $0.07(0.02-0.33)$ & $0.20(0.00-0.60)$ & 0.81 \\
DNASE1L3 expression & $0.26(0.11-0.50)$ & $0.39(0.23-5.3)$ & 0.91 \\
IL1B expression & $52.6(32.2-107.0)$ & $114(49.5-246.4)$ & 0.77 \\
ALPL expression & $1.8(0.62-4.83)$ & $4.84(1.80-9.34)$ & 0.74 \\
CXCR2 expression & $5.63(3.66-24.3)$ & $12.32(6.93-19.0)$ & 0.83
\end{tabular}

Data are presented as median (interquartile range) unless otherwise stated. Gene expression presented as cycle threshold change $\left(2^{-\Delta C T}\right)$ multiplied by 10000 . 

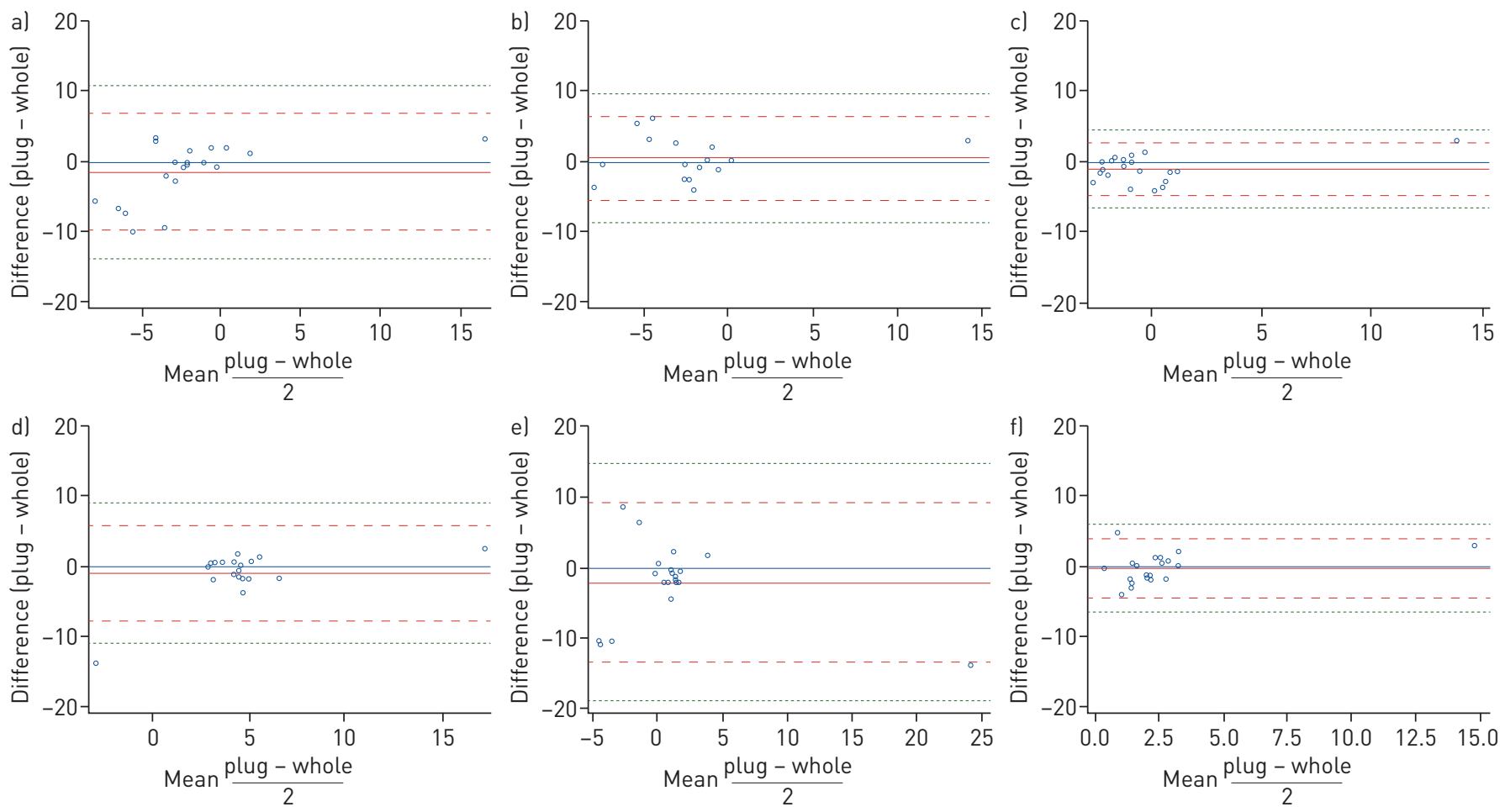

FIGURE 2 Bland-Altman plots of gene expression. a) CLC, b) CPA3, c) DNASE1L3, d) IL1B, e) $A L P L$ and f) CXCR2. Blue: ref.; solid red: mean of the difference; dashed red: limits of agreement; dashed green: $\pm 3 \times S D$ of the mean of the differences.

from patients with non-eosinophilic asthma. Despite this, discrimination was only significant for the whole sampled sputum and we did not find a statistically significant difference between the two ROC curves $(\mathrm{p}=0.14)$.

We found fair agreement (kappa coefficients of $0.36(\mathrm{p}<0.001))$ for discriminating eosinophilic asthma from non-eosinophilic asthma using 6GS for the manually selected plugs and a substantial agreement (kappa coefficient $0.68(\mathrm{p}<0.001))$ for the whole sampled sputum when comparing to gold-standard classification based on the CDC (table 3) [21, 22].

The whole sampled sputum had a higher sensitivity, specificity and both PPV and NPV than the manually selected plugs (table 3 ). Comparing the plugs selected and whole sample method directly gave a kappa coefficient of $0.67(\mathrm{p}<0.001)$.
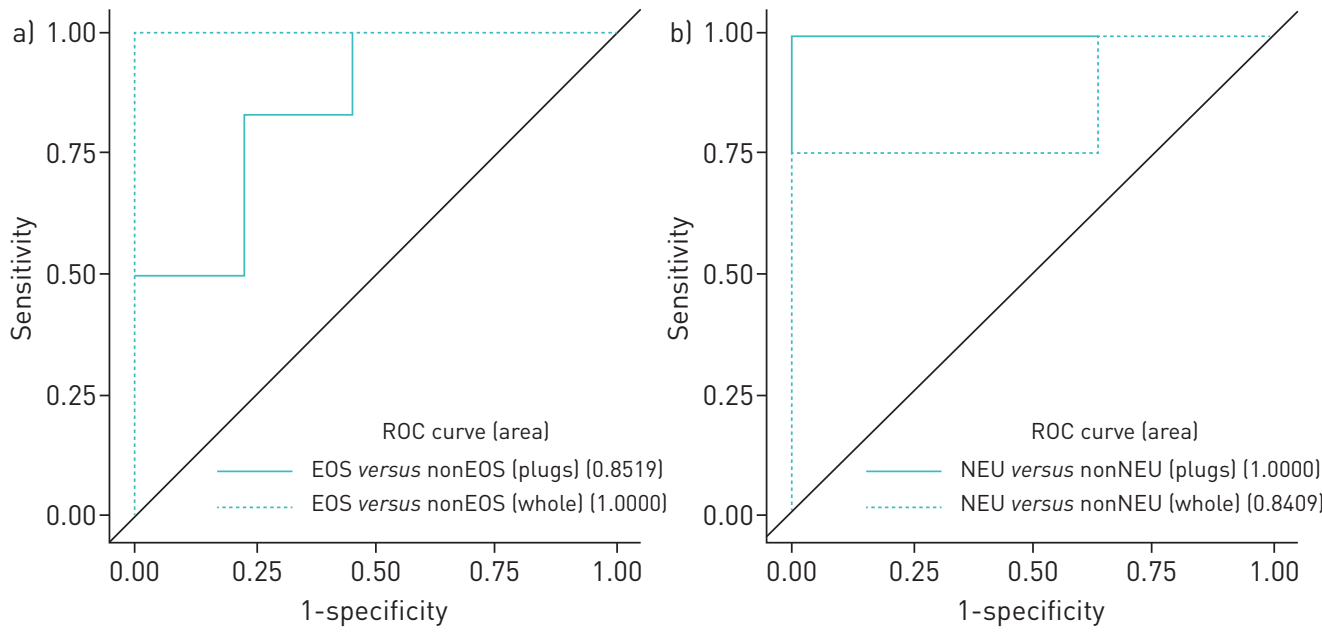

FIGURE 3 Receiver operating characteristic (ROC) curves for predicting inflammatory phenotype using the six-gene signature. a) Eosinophilic (EOS) asthma versus non-eosinophilic (nonEOS) asthma. b) Neutrophilic (NEU) asthma versus non-neutrophilic (nonNEU) asthma. 


\begin{tabular}{|c|c|c|c|c|c|c|c|c|}
\hline & AUC & p-value & Cut-off point & Sens & Spec & PPV & NPV & Kappa \\
\hline \multicolumn{9}{|c|}{ Eosinophilic asthma versus non-eosinophilic asthma } \\
\hline Manual plugs & 0.85 & 0.1 & 0.39 & 56 & 80 & 71 & 67 & 0.22 \\
\hline Whole sample & 1.00 & 0.01 & 0.99 & 67 & 100 & 100 & 77 & 0.72 \\
\hline \multicolumn{9}{|c|}{ Neutrophilic asthma versus non-neutrophilic asthma } \\
\hline Manual plugs & 1.00 & 0.008 & 0.99 & 80 & 100 & 100 & 93 & 0.68 \\
\hline Whole sample & 0.84 & 0.2 & 0.62 & 80 & 100 & 100 & 93 & 0.68 \\
\hline \multicolumn{9}{|c|}{$\begin{array}{l}\text { All measures are relative to the classification from the manual cell differential count. AUC: area under the } \\
\text { curve; Sens: sensitivity; Spec: specificity; PPV: positive predictive value; NPV: negative predictive value; } \\
\text { Kappa: Cohen's kappa coefficient for agreement with the gold-standard classification based on the cell } \\
\text { differential count. }\end{array}$} \\
\hline
\end{tabular}

Discriminating neutrophilic asthma from non-neutrophilic asthma

The 6GS resulting from manually selected plugs (figure 3b; AUC 100\%, p=0.008) and whole sampled sputum (figure $3 \mathrm{~b}$; AUC 85\%, $\mathrm{p}=0.2$ ) was able to discriminate patients with neutrophilic asthma from patients with non-neutrophilic asthma. Despite this, discrimination was only significant for the manually selected plugs and we did not find a statistically significant difference between the two ROC curves $(\mathrm{p}=0.33)$.

We found substantial agreement in discrimination of neutrophilic asthma from non-neutrophilic asthma using both methods compared with the gold-standard classification based on the CDC ("plug selection method": kappa coefficient of 0.85 ( $\mathrm{p}<0.001)$; "whole sputum method": kappa coefficient of 0.85 $(\mathrm{p}<0.001))($ table 3) $[21,22]$.

The two methods had identical sensitivity, specificity and PPV and NPV (table 3). Comparing the plugs and whole sample method directly gave a kappa coefficient of $0.69(\mathrm{p}<0.001)$.

\section{Discussion}

We found sputum collected using the "whole sputum method" in an mRNA-stabilising kit to yield more RNA and have a consistently higher level of gene expression across all genes compared with sputum processed using the "plug selection method". Further, we found the two methods to agree and to have a good-to-excellent reliability [20,23]. Finally, expression of the 6GS using both methods performed well in the identification of the inflammatory phenotypes, although the sample collected using the "whole sputum method" in the mRNA-stabilising kit had an improved detection of eosinophilic inflammation.

Compared to our previous work using the 6GS ("plug sputum method") to discriminate inflammatory phenotypes, we found the 6GS to be similarly accurate in discriminating inflammatory phenotypes of asthma using both of the assessed methods [5]. However, as the study was powered to compare gene expression levels rather than discriminate inflammatory phenotypes, these results are limited by the small sample size and future studies validating these findings are warranted.

While the "plug sputum method" is employed by most research groups assessing mRNA expression in sputum [5-7], no other studies have reported analysis of mRNA in sputum collected using the "whole sputum method". However, the two methods of sputum collection, despite only few studies comparing them directly in terms of differential cell count, have been found to yield similar results, although a higher viability of cells has been reported using the "whole sputum method" [4, 24].

Seventy-five percent $(n=20)$ of the included patients had sufficient quality of samples (figure 1) to be included in the analysis of RNA yield and comparison of mRNA expression. Of these, 18 had a plug enabling a CDC (figure 1). While exclusion of $33 \%$ of patients due to insufficient sputum samples is unfortunate, and may have introduced a selection bias, we do not believe that it reflects a poor external validity as this issue is universally recognised, as exemplified by just $44 \%$ of sputum samples fit for analysis in the U-BIOPRED cohort [25]

Sputum for both processing methods was collected on the same day and after only one induction. We chose this approach, rather than collecting each sample after a separate induction, based on the reports of changes in sputum cellularity after repeated sputum induction within up to $48 \mathrm{~h}[26,27]$. While it might have been optimal to collect the samples after separate inductions on different days, as the differential cell count has been reported to be reproducible within a week using both the "whole sputum method" [28] and the "plug selection method" [29], we chose to do it on the same day due to practicality. As such, 
we may have introduced a bias, given the fixed order of sample collection; the first sample (collected using the "plug selection method") may be subject to more contamination from the upper airways than the second sample.

We found a higher RNA yield and a consistently higher level of gene expression across all genes in the sputum collected using the "whole sputum method" in an mRNA-stabilising kit. This may to some extent reflect the larger amount of sample collected using the "whole sputum method", including contamination with saliva $[3,4]$. However, the standardisation relative to a housekeeping gene should mitigate this effect and we believe that the immediate stabilisation of the sample in an mRNA-stabilising buffer is the main reason for this difference. In contrast, the sample processing using the standard "plug selection method" protocol may be stored for up to $2 \mathrm{~h}$ before processing, which in turn may result in a significant degradation of mRNA.

Our findings demonstrate that sputum collected using the "whole sputum method" and immediately stabilised using an mRNA-stabilising kit is a reliable method for inflammatory phenotyping that yields comparable or even superior results, compared to the "plug selection method" using a standard sputum processing protocol. As collection of sputum using this method requires neither immediate handling nor laboratory facilities at the site of collection, it could potentially provide inflammatory phenotyping of asthma at a much larger scale than is currently possible. Further, it provides a feasible method for biobanking of airway samples in clinical trials and could be pivotal in large-scale multicentre studies, where the logistical challenges of the manual plug selection may impede its use. Similarly, it could play a key role in facilitating clinically feasible inflammatory phenotyping and biobanking in the increasing number of severe asthma registries, which represent unique prospective real-life cohorts of patients where targeted therapies are initiated, evaluated and interchanged.

In conclusion, sputum collected using the "whole sputum method" in an RNA-stabilising kit is a simple method that obviates the need for immediate handling and provides a clinically feasible method for inflammatory phenotyping and biobanking in clinical care, as well as in large-scale clinical studies.

Further, our findings warrant future studies comparing the "whole sputum method" and "the plug selection method" for gene expression analysis; both with and without the use of RNA-stabilising kits.

Conflict of interest: L. Frøssing has nothing to disclose. D. Kjærsgaard Klein has nothing to disclose. V. Backer has nothing to disclose. K.J. Baines reports patent PCT/AU2013/001466 6GS in asthma issued and patent PCT/AU2018/ 050644 6GS in COPD pending. C. Porsbjerg has nothing to disclose.

\section{References}

1 Chung KF, Wenzel SE, Brozek JL, et al. International ERS/ATS guidelines on definition, evaluation and treatment of severe asthma. Eur Respir J 2014; 43: 343-373.

2 Hamid Q, Kelly MMM, Linden M, et al. Methods of sputum processing for cell counts, immunocytochemistry and in situ hybridisation. Eur Respir J 2002; 20: Suppl. 37, 19S-23S.

3 Gershman NH, Wong HH, Liu JT, et al. Comparison of two methods of collecting induced sputum in asthmatic subjects. Eur Respir J 1996; 9: 2448-2453.

4 Pizzichini E, Pizzichini MMM, Efthimiadis A, et al. Measurement of inflammatory indices in induced sputum: effects of selection of sputum to minimize salivary contamination. Eur Respir J 1996; 9: 1174-1180.

5 Baines KJ, Simpson JL, Wood LG, et al. Sputum gene expression signature of 6 biomarkers discriminates asthma inflammatory phenotypes. J Allergy Clin Immunol 2014; 133: 997-1007.

6 Truyen E, Coteur L, Dilissen E, et al. Evaluation of airway inflammation by quantitative Th1/Th2 cytokine mRNA measurement in sputum of asthma patients. Thorax 2006; 61: 202-208.

7 Kuo C-HS, Pavlidis S, Loza M, et al. T-helper cell type 2 (Th2) and non-Th2 molecular phenotypes of asthma using sputum transcriptomics in U-BIOPRED. Eur Respir J 2017; 49: 1602135.

8 Pavord ID, Pizzichini MM, Pizzichini E, et al. The use of induced sputum to investigate airway inflammation. Thorax 1997; 52: 498-501.

9 Wood LG, Powell H, Gibson PG. Mannitol challenge for assessment of airway responsiveness, airway inflammation and inflammatory phenotype in asthma. Clin Exp Allergy 2010; 40: 232-241.

10 Alvarez-Puebla M, Olaguibel J, Almudevar E, et al. Mannitol versus hypertonic saline. Chron Respir Dis 2015; 12: 197-203.

11 Bafadhel M, McCormick M, Saha S, et al. Profiling of sputum inflammatory mediators in asthma and chronic obstructive pulmonary disease. Respiration 2012; 83: 36-44

12 DNA Genotek. 2012. Oragene ${ }^{\circledast}$ RNA purification protocol using the Qiagen RNeasy Micro Kit for volumes up to $1000 \mu \mathrm{L}$. https://www.dnagenotek.com/ROW/pdf/PD-PR-021.pdf

13 Schmittgen TD, Livak KJ. Analyzing real-time PCR data by the comparative CT method. Nat Protoc 2008; 3: $1101-1108$.

14 Bousquet J, Khaltaev N, Cruz AA, et al. Allergic rhinitis and its impact on asthma (ARIA) 2008. Allergy 2008; 63: $8-160$.

15 Miller MR, Hankinson J, Brusasco V, et al. Standardisation of spirometry. Eur Respir J 2005; 26: 319-338.

16 Hankinson JL, Odenkrantz JR, Fedan KB. Spirometric reference values from a sample of the general U.S population. Am J Respir Crit Care Med 1999; 159: 179-187. 
17 ATS/ERS. ATS/ERS recommendations for standardized procedures for the online and offline measurement of exhaled lower respiratory nitric oxide and nasal nitric oxide, 2005. Am J Respir Crit Care Med 2005; 171: 912-930.

18 Bland JM, Altman D. Statistical methods for assessing agreement between two methods of clinical measurement. Lancet 1986; 327: 307-310.

19 Baines KJ, Simpson JL, Wood LG, et al. Transcriptional phenotypes of asthma defined by gene expression profiling of induced sputum samples. J Allergy Clin Immunol 2011; 127: 153-160.

20 Koo TK, Li MY. A guideline of selecting and reporting intraclass correlation coefficients for reliability research. J Chiropr Med 2016; 15: 155-163.

21 Landis JR, Koch GG. The measurement of observer agreement for categorical data. Biometrics 1977; 33: 159-174.

22 McHugh ML. Interrater reliability: the kappa statistic. Biochem Med (Zagreb) 2012; 22: 276-282.

23 Cicchetti DV. Guidelines, criteria, and rules of thumb for evaluating normed and standardized assessment instruments in psychology. Psychol Assess 1994; 6: 284-290.

24 Pizzichini E, Pizzichini MM, Gibson P, et al. Sputum eosinophilia predicts benefit from prednisone in smokers with chronic obstructive bronchitis. Am J Respir Crit Care Med 1998; 158: 1511-1517.

25 Shaw DE, Sousa AR, Fowler SJ, et al. Clinical and inflammatory characteristics of the European U-BIOPRED adult severe asthma cohort. Eur Respir J 2015; 46: 1227-1231.

26 Nightingale JA, Rogers DF, Barnes PJ. Effect of repeated sputum induction on cell counts in normal volunteers. Pneumologie 1998; 52: 400-401.

27 Van Der Vaart H, Postma DS, Timens W, et al. Repeated sputum inductions induce a transient neutrophilic and eosinophilic response. Chest 2006; 130: 1157-1164.

28 Bacci E, Cianchetti S, Carnevali S, et al. Induced sputum is a reproducible method to assess airway inflammation in asthma. Mediators Inflamm 2002; 11: 293-298.

29 Spanevello A, Migliori GB, Sharara A, et al. Induced sputum to assess airway inflammation: a study of reproducibility. Clin Exp Allergy 1997; 27: 1138-1144. 